\title{
Informed Consent in the Health Care System: An Overview from a Dental Perspective in Saudi Arabia
}

\author{
Afnan A. Nassar ${ }^{a} \quad$ Abrar K. Demyati ${ }^{b}$ \\ ${ }^{a}$ Department of Preventive Dentistry at the Faculty of Dentistry, Division of Dental Public Health, \\ Umm Al-Qura University, Makkah, Saudi Arabia; ${ }^{\mathrm{b}}$ Department of Oral and Maxillofacial Surgery and Diagnostic \\ Sciences, Division of Oral and Maxillofacial Surgery, Umm Al-Qura University, Makkah, Saudi Arabia
}

\section{Keywords}

Autonomy · Dentistry · Ethics · Informed consent ·

Saudi Arabia

\begin{abstract}
Background: Patient autonomy in the health care system is achieved by the vital principle of providing informed consent. Throughout history, informed consent gained recognition and improved to include more aids and steps to formalize and standardize the process of obtaining proper consent in medical and dental practice. Regardless of the type of informed consent obtained before the treatment, it should include an adequate understandable description of nature and diagnosis of the disease, treatment plan, proper alternatives, risks, and limitations. Summary: There is limited information in the ethics literature covering critical concepts related to different dental procedures in Saudi Arabia. In Saudi Arabia, informed consent in dentistry is not well-documented. As everything is evolving and changing in Saudi society, litigation has progressed and impacted dentistry. This overview will help in addressing aspects related to informed consent and closing the gaps in the dental health care system in Saudi Arabia, managing complex ethical issues associated with dental patients. In addition, providing recommendations and shedding some light on the importance of in-
\end{abstract}

karger@karger.com www.karger.com/sjh

Karger $\frac{1}{\%}$

GOPEN ACCESS
(C) 2021 The Author(s)

Published by S. Karger AG, Basel

This article is licensed under the Creative Commons AttributionNonCommercial-NoDerivatives 4.0 International License (CC BYNC-ND) (http://www.karger.com/Services/OpenAccessLicense). Usage and distribution for commercial purposes as well as any distribution of modified material requires written permission. formed consent will improve the situation of the informed consent process in Saudi Arabia. Key Messages: Informed consent allows patients to be part of the decision-making process, and it provides legal protection for the practitioners from practice lawsuit cases. Dentists should take extra care in documenting the consent process and patient's choice regarding their treatment to avoid unfavorable consequences. In Saudi Arabia, attention should be drawn toward the crucial role of informed consent, and more studies should be published in order to enrich the knowledge and to improve the health care system.

(C) 2021 The Author(s)

Published by S. Karger AG, Basel

\section{History of Informed Consent}

The Hippocratic principles (400-300 BC) established the first medical ethics [1]. For centuries, only beneficence, nonmaleficence, and confidentiality played a fundamental role in the ethics and conducting a health-care intervention on a person [1]. Hippocrates has allowed doctors to decide in a parental fashion the patient's best interest [2].

The earliest expression of informed consent and autonomy is found in the Nuremberg Code of ethics in 1947 after World War II in response to the unethical medical 
experiments performed by the Nazi doctors [2]. The code mandates to obtain the voluntary informed consent of human subjects [2]. Similarly, in 1964, the Declaration of Helsinki by the World Medical Association emphasizes the importance of attaining freely voluntary informed consent for medical research [2].

In dentistry, obtaining informed consent from patients was introduced in the mid-1980s, and since then, this process shifted from a paternalistic-focused model to a patient autonomy-focused model [3]. Nowadays, informed consent plays an integral part in routine dental practice, which allows patients to raise concerns at any point about their treatment options [4]. Several additional aids have been added to improve informed consent in dentistry such as incorporating leaflets, multimedia, and shared decision-making method where patients are given high-quality information that allows them to participate actively in making decisions about their treatment. These methods produce more positive outcomes than the conventional standard method $[5,6]$.

\section{Informed Consent: What and Why}

Informed consent is a series of proceedings and not done by only obtaining a signature on a paper [7]. It is the process of constructive, informative discussion between the treatment provider and the patient throughout the treatment [7]. According to the British Dental Association, informed consent is considered when the patient agrees to receive an examination or a treatment either orally or in writing or for personal information to be processed [8]. The main aim of the consent process is to provide the patients with enough information to allow them to preserve their right to understand their options and make an informed decision about their health condition [8]. Nowadays, patient consent should be obtained before every medical or dental procedure [8].

Nevertheless, it is equally important for health providers to officially record patient consent to protect themselves from illegal or malpractice issues [9]. Laws concerning informed consent differ from country to country, and also the patient's perception of medical errors and expectations of treatment may differ from one generation to another [9]. Informed consent involves a comprehensive description of the nature and diagnosis of the condition and purpose and material of the planned treatment using simple terms that the patient comprehends, also risks, benefits, alternatives, and limitations [7, 9]. Patients must have the chance to reflect on their opinion and ask about the received information to allow a sensible decision of whether to continue with the proposed treatment or not $[7,9]$. The consent must be valid and specific and given by a responsible adult patient or a parent or a guardian before starting any treatment $[7,9]$.

From a dentist's perspective regarding obtaining consent in dental practice, most dentists applied informed consent before performing any dental procedures [10]. A Bulgarian study found that most dentists took consent while treating children but when treating friends, relatives, and longtime patients, they were less careful in taking consent [11].

From a patient's perspective, a study used patient-directed questionnaire showed that most patients were unaware that the consent process serves their interests by allowing them to choose their treatment voluntarily [12]. Also, evidence shows that after the process of informed consent, patients did not always understand or recall the information provided for the proposed treatment and associated risks and benefits [6].

\section{Types of Informed Consent}

\section{Implied Consent}

This type of consent reflects passive cooperation in the course of treatment without going through formal consent steps [9]. It mainly depends on proper communication between the health provider and the patient. It provides all the needed information to allow the patient to understand the process and its rationale. Implied consent documentation in the clinical record is not required [9]. An implied consent example is when the patient is lying in the dental chair and opening the mouth for examination [7].

\section{Verbal Consent}

It is verbal communication between the provider and the patient, where the patient agrees to the suggested procedure without any signature $[7,9]$. It is satisfactory for any routine examination such as diagnostic and prophylactic procedures $[7,9]$. It is required to register all the steps done in medical records $[7,9]$. The Australian Dental Association states that oral consent is adequate for most dental procedures, but a written consent form is recommended for major treatment, either in terms of invasiveness or expense [13]. Informed consent should be clear to allow the patient to voluntarily agree to the treatment [14].
Nassar/Demyati 


\section{Written Consent}

This form is mandatory in extensive treatment involving risks where anesthesia, tooth preparations, surgical procedures, implant placement, extractions, and administration of medications with known side effects $[7,9]$. Signature is required in such procedures $[7,9]$.

\section{Consent and Legal Matters}

Over the last decade, dental practice lawsuits increased when patients were unhappy with the treatment provided, and they felt that treatment options were not properly discussed [15]. In particular, if the final clinical outcomes differ from the patient's expectations because it was not clear from the beginning, patients may request legal help to address their complaints [15-17]. A study in Brazil surveyed 179 dentists working in operative dentistry providing aesthetic restorations showed that about $3 \%$ of the study population faced legal issues [18]. The 3\% complaints were related to dentists practicing while not being completely aware of dental legal matters [18]. These legal concerns in operative dentistry could be avoided if routine ethical practice including informed consent were obtained [18].

Another example in the field of oral surgery where a study investigated 225 patients presenting to an emergency clinic seeking oral surgical treatment. This study reported that $10 \%$ of the study population did not have interest to know about potential complications, while $57 \%$ of the patients desired to know about all possible complications [9]. It is not reliable to depend on patient's memory when it comes to treatment plan and legal consequences. It has been reported that patients believed that they did not receive any information, while few patients could remember complications explained in regard to their treatment options [4]. Carefully constructed informed consent form protects dentists from litigation and allows patients to learn about different procedures and associated risks and complications and decide accordingly [9].

It is advised to rely on written consent as a legal evidence and/or to document verbal consent in the medical records. This recommendation comes from reviewing the evidence that patients did not always understand the information provided by the treating dentist, while dentists believed they understood [4]. Dentist's awareness is crucial; a study included 100 dentists who filled an online survey showed that only $78 \%$ of the participants knew about the importance of the informed consent medicolegal aspects [19].

Consent in Dentistry

\section{Consent in Saudi Arabia}

In Saudi Arabia, clinical practice used informed consent that has been translated and acquired from Western countries [20]. Due to cultural idiosyncrasies, Western policy informed consent were not entirely applicable in Saudi Arabia, where culture gives the ultimate trust to the physician without the need for high-context communication and autonomy is not the first patient concern [21]. It has been claimed that explaining details to patient may raise doubts and suspicions about the treatment [22]. However, a study about information disclosure in clinical informed consent in a tertiary health-care hospital in Saudi Arabia stated that $70.0-98.0 \%$ of their respondents preferred to have most of the details associated with the procedures disclosed by the operators [23]. The Saudi Council for Health Specialties and the Saudi Arabian Ministry of Health as a part of the medical profession's bioethics composed guidelines for informed consent process [24, 25]. The guidelines were acquired from internationally established good practice policy combined with crucial social representation of the Saudi culture, and Islamic law states that "prior to delivering medical treatment or carrying out an operative procedure, the legally competent patient's consent, be he/she male or female, shall be obtained," and "the physician shall provide an adequate explanation to the patient or his guardian on the nature of the medical treatment or operative procedure he intends to apply" [24, 25].

A study was done at King Abdulaziz Medical City (KAMC), Riyadh, Saudi Arabia, to assess the quality of informed consent for invasive procedures from patient's point of view [20]. It showed that more than half of the study population believed that their choice was unnecessary because the operator had decided for them [20]. Another study from the Ministry of Health, Dental Department in Riyadh city, demonstrated malpractice lawsuit cases, which arose in Riyadh throughout 1 year (1997) [26]. It was reported that 32 dental complaints were filed, 20 cases had clinical issues and 12 were nonclinical cases [26]. Regarding the clinical complaints, $18.8 \%$ were related to oral surgery, with $15.6 \%$ in fixed prosthodontics procedures [26]. The alarming finding in this previous study was that only 1 case had an informed consent documented [26]. There is little information published related to ethical standards for different dental procedures in Saudi Arabia. Addressing these aspects can help close the gaps and improve the quality of the dental health care system in managing complex ethical issues associated with dental patients. 


\section{Content of Informed Consent according to Saudi Requirement}

According to the Saudi Guide from the Ministry of Health, the consent form for dental treatment should be in both Arabic and English languages [24]. It must include the following: symbol of the dental institution providing the treatment, details about the name of the institution, the name of the department where the treatment is taking place, the name of the dentist managing the case, paper heading with the consent type written, description of the procedure with all possible risks, date and time, and a signature from both the patient and the dentist [24].

\section{Recommendations}

Dentists need to be familiar with the concept of recorded informed consent in the medical records, even if not a signed format, as it is one of the essential legal protections and ethical responsibilities of dentists. For the consent form to be consistent, we advise having a consent template for all the different procedures in dentistry (surgery, endodontics, periodontics, and prosthodontics), with all the required simple information ready to be handed to the patients rather than having a general consent that is only taken in the event of invasive dental procedures. To help in the consent process, we suggest having standardized consent forms prepared by Saudi experts in each dental division that can be downloaded from the Ministry of Health website would be the ultimate goal to improve the informed consent process in Saudi Arabia. Incorporation of ethical education for dental students and it should focus on the legal issues of properly obtaining informed consent among their studies and later when practicing dentistry. Our recommendation also extends to patients. Before obtaining informed consent, information regarding dental procedures might be delivered in various formats to ensure patients understanding such as paper, videos, and pictures. The need for more work that provides patients' awareness of their rights and the acceptability of all different sorts of information emphasizes the importance of reevaluating the consent form process in the Saudi population.

\section{Conclusion}

Informed consent is essential in the dental practice and ethics in international as well as the Saudi national guidelines. As a health care provider, we hope that this work will enlighten the practice of using informed consent and its role in bioethics and encourage good clinical practice ethical standards.

\section{Acknowledgement}

The authors would like to acknowledge the support from the Faculty of Dentistry, Umm Al-Qura University.

\section{Statement of Ethics}

IRB approval was not required for this review, and the Faculty of Dentistry approved the topic.

\section{Conflict of Interest Statement}

There are no conflicts of interest.

\section{Funding Sources}

The authors did not receive any funding.

\section{Author Contributions}

Both authors have contributed equally in planning, reviewing, and writing this paper.

\section{References}

1 Sauer JE Jr. Ethical problems facing the healthcare industry. Hosp Health Serv Adm. 1985;30(5):44-53.

2 Al-Bar MA, Chamsi-Pasha H. Regulation of medical profession and medical research. Contemporary bioethics: Islamic perspective. Cham: Springer International Publishing; 2015. p. 85-103.
3 Reid KI. Informed consent in dentistry. J Law Med Ethics. 2017;45(1):77-94.

4 Seldin LW. Informed consent. The patient's rights. Dent Today. 2003;22(12):86-8; quiz 9; Holden A, Stewart C. A review of the law of consent in dentistry. Aust Dent J. 2019;64(3): $208-12$.
5 Asa'ad F. Shared decision-making (SDM) in dentistry: a concise narrative review. J Eval Clin Pract. 2019;25(6):1088-93.

6 Moreira NC, Pachêco-Pereira C, Keenan L, Cummings G, Flores-Mir C. Informed consent comprehension and recollection in adult dental patients: a systematic review. J Am Dent Assoc. 2016;147(8):605-19.e7. 
7 Anil Sharma AC, Sharma A, Bopiah C. Patient consent in dentistry: are we legally safe? J Oral Health Comm Dent. 2011;5(2):68-72.

8 British Dental Association. Ethics in dentistry. British Dental Association; 2000.

9 Degerliyurt K, Gunsolley JC, Laskin DM. Informed consent: what do patients really want to know? J Oral Maxillofac Surg. 2010;68(8): 1849-52.

10 Dhingra A, An R. Consent in dental practice: patient's right to decide. J Oral Hyg Health. 2014;2:1-5.

11 Avramova N, Yaneva K. Patients' informed consent in dental practice in Bulgaria. OHDM. 2011;10(2):80-7.

12 Hajivassiliou EC, Hajivassiliou C. Informed consent in primary dental care: patients' understanding and satisfaction with the consent process. Br Dent J. 2015;219(5):221-4.

13 Australian Dental Association. Policy statement 5.15.: consent to treatment, ADA guidelines to consent to dental treatment. 2020. [cited 2020 November 10th]. Available from: https: //www.ada.org.au/Dental-Professionals/Policies/Third-Parties/5--15-Consent-to-
Treatment/ADAPolicies_5--15_ConsenttoTreatment_V1.aspx.

14 de Medeiros UV, Coltri AR. Responsabilidade civil do cirurgião-dentista. Rev Bras Odontol. 2014;71(1):10-6.

15 Lam R. Minimally legally invasive dentistry. Aust Dent J. 2014;59(4):432-8.

$16 \mathrm{Bal} \mathrm{BS}$. An introduction to medical malpractice in the United States. Clin Orthop Relat Res. 2009;467(2):339-47.

17 Jena AB, Seabury S, Lakdawalla D, Chandra A. Malpractice risk according to physician specialty. N Engl J Med. 2011;365(7):629-36.

18 Rodrigues LG, De Souza JB, De Torres EM, Silva RF. Screening the use of informed consent forms prior to procedures involving operative dentistry: ethical aspects. J Dent Res Dent Clin Dent Prospects. 2017;11(1):66.

19 Akkad A, Jackson C, Kenyon S, DixonWoods M, Taub N, Habiba M. Patients' perceptions of written consent: questionnaire study. BMJ. 2006;333(7567):528.

20 Abolfotouh MA, Adlan AA. Quality of informed consent for invasive procedures in central Saudi Arabia. Int J Gen Med. 2012;5:269.
21 Del Pozo PR, Fins JJ. Islam and informed consent: notes from Doha. Camb Q Healthc Ethics. 2008;17(3):273.

22 Adlan AA. Informed consent in Saudi Arabia. Forensic Leg Med. 2013:893-907.

23 Hammami MM, Al-Jawarneh Y, Hammami $\mathrm{MB}, \mathrm{Al}$ Qadire M. Information disclosure in clinical informed consent: "reasonable" patient's perception of norm in high-context communication culture. BMC Med Ethics. 2014;15(1):3.

24 Ministry of Health. Saudi directory of medical consent. 2019; [cited 2020 Nov 10]. Available from: https: //www.moh.gov.sa/Documents/2019--12--09--001.pdf.

25 Saudi Commision for Health Specialties. Code of ethics for healthcare practitioners. 2014; [cited 2020 Nov 10]. Available from: https: //www.scfhs.org.sa/en/Media/OtherPublications/Documents/Professionalism $\% 20$ and $\% 20$ Ethics\%20Handbook\%20 for\%20Residents.pdf.

26 Al-Ammar W, Guile EE. A one-year survey of dental malpractice claims in Riyadh. Saudi Dent J. 2000;12(2):95-9. 